\title{
Monitoring Tool Wear Using Classifier Fusion
}

\author{
Elijah Kannatey-Asibu \\ Professor \\ Department of Mechanical Engineering \\ The University of Michigan \\ Ann Arbor, MI 48109-2125 \\ E-mail: asibu@umich.edu \\ Tel: 734-945-5560 \\ Juil Yum \\ Graduate Student Research Assistant \\ Department of Mechanical Engineering \\ The University of Michigan \\ Ann Arbor, MI 48109-2125 \\ E-mail: yumjuil@umich.edu \\ T.H. Kim \\ Assistant Research Scientist \\ Department of Mechanical Engineering \\ The University of Michigan \\ Ann Arbor, MI 48109-2125
}

\begin{abstract}
Real time monitoring of manufacturing processes using a single sensor often poses significant challenge. Sensor fusion has thus been extensively investigated in recent years for process monitoring with significant improvement in performance. This paper presents the results for a monitoring system based on the concept of classifier fusion, and class-weighted voting is investigated to further enhance the system performance. Classifier weights are based on the overall performances of individual classifiers, and majority voting is used in decision making. Acoustic emission monitoring of tool wear during the coroning process is used to illustrate the concept. A classification rate of $87.7 \%$ was obtained for classifier fusion with unity weighting. When weighting was based on overall performance of the respective classifiers, the classification rate improved to $95.6 \%$. Further using state performance weighting resulted in a $98.5 \%$ classification. Finally, the classifier fusion performance further increased to $99.7 \%$ when a penalty vote was applied on the weighting factor.
\end{abstract}

Keywords: acoustic emission, classifier fusion, coroning, tool wear monitoring, weighted voting 


\title{
Monitoring Tool Wear Using Classifier Fusion
}

\begin{abstract}
Real time monitoring of manufacturing processes using a single sensor often poses significant challenge. Sensor fusion has thus been extensively investigated in recent years for process monitoring with significant improvement in performance. This paper presents the results for a monitoring system based on the concept of classifier fusion, and class-weighted voting is investigated to further enhance the system performance. Classifier weights are based on the overall performances of individual classifiers, and majority voting is used in decision making. Acoustic emission monitoring of tool wear during the coroning process is used to illustrate the concept. A classification rate of $87.7 \%$ was obtained for classifier fusion with unity weighting. When weighting was based on overall performance of the respective classifiers, the classification rate improved to $95.6 \%$. Further using state performance weighting resulted in a $98.5 \%$ classification. Finally, the classifier fusion performance further increased to $99.7 \%$ when a penalty vote was applied on the weighting factor.
\end{abstract}

Keywords: acoustic emission, classifier fusion, coroning, tool wear monitoring, weighted voting 


\section{INTRODUCTION}

The difficulty associated with tool condition monitoring during machining is primarily due to thecontinuousnature of tool wear. Several techniques have thus been investigated for estimating the tool condition [1-6]. A variety of classifiershave also been used for this purpose, and these include artificial neural networks [4, 7-11], hidden Markov model[12,13],k-nearest neighbor [14], maximum likelihood, and support vector machine [15].

Since individual classifiers perform differently, depending on the type of application, the use of multiple classifiers has been investigated in some fields for making monitoring systems more robust [16-22]. Conceptually, this is similar to sensor fusion which capitalizes on the advantages of individual sensors and reduces sensitivity to noise [8, 23-27]. Classifier fusion, on the other hand, capitalizes on the advantages of individual classifiers.

In earlier work on classifier fusion [30], a technique was investigated that evaluates the performances of a number of classifiers and selects the best among them using the concept of "overproduce and choose". This is similar in concept to the class mean scatter (CMS) criterion [31], which is based on ranking of candidate process features.

In an application based on the modified Bagging method, the best of several artificial neural networks was selected for predicting the state of tool wear during drilling [32]. Another method for monitoring drilling operations was based on a decision fusion center algorithm [33]. Monitoring of the end milling process has also been investigated using machine ensemble techniques such as majority voting and generalized stacking [34].

Multi-classifier algorithms have often made use of a voting system, for example, majority voting [35, 36]. However, as pointed out by Petrakoset al.[37], the results obtained using classifier fusion will not differ from that of a single classifier if the classifiers agree on class 
decision. On the other hand, voting plays an important role if the individual classifiers have different decisions. Another major challenge associated with voting is the issue of 'tie votes'. This is often addressed using weighted voting $[38,39]$ where the weights are normally constant. In situations where the classifier performances vary in the course of the process, such as may occur during tool degradation, then it becomes essential to account for such variation.

This paper extends the concept of decision fusion to classifiers, with a view to making tool condition monitoring systemsmore robust. This would enhance their performance by reducing classification errors that may result with individual classifiers. In addition,the state-performance weighting factor and penalty voting concepts are introduced to further improve classification rates.

Acoustic emission (AE) is used as the sensor signal, and the process investigated is the coroning process. Thus in section 2, a brief background is provided on AE, the coroning process, and classifier fusion. This is followed in section 3 by the experimental procedure. The results are presented and discussed in section 4, and finally, the conclusions in section 5.

\section{BACKGROUND}

\subsection{Acoustic Emission (AE)}

Acoustic emission refers to the elastic stress waves generated as a result of the rapid release of strain energy within a material due to a re-arrangement of its internal structure. Early applications of $\mathrm{AE}$ to machining can be traced to the work of Grabec and Leskovar[40], and Iwata and Moriwaki[41], who examined the fundamental characteristics of AE from machining. Subsequent work by Moriwaki[42] indicated that AE signals with large amplitude were associated with tool failures such as cracking, chipping, and fracture. Kannatey-Asibu and 
Dornfeld[43] later developed a relationship between AE and the cutting process. Good correlation was found between predicted and experimental results.

Emel and Kannatey-Asibu[44] monitored tool wear and breakage using pattern recognition analysis ofAE signals generated during the process. In order to reduce cutting condition effects, an autoregressive analysis was used to model the acoustic emission signal sensed from the cutting process by Liang and Dornfeld[45]. Teti [46] presented experimental results for AE generation during machining of carbon steel using high speed steeltools under realistic cutting conditions. Blum and Inasaki[47] investigated both the force and AE signal generation during orthogonal cutting. A neural network consisting of two sequential learning stages, unsupervised Kohonen's feature map and input feature scaling was introduced by Leem et al. [4] for on-line monitoring of tool wear. High accuracy rates with robustness in the classifications of time and three levels of tool wear were achieved. In another application, AE was used to monitor both chatter and tool wear by Chiou and Liang [48].

A comprehensive summary of early work on AE monitoring of the machining process was presented in a review by Dornfeld[49] and Li [50]. Recent research in this field has focused more on micromachining operations[51-53]. Hung and $\mathrm{Lu}$ [53] modeled $\mathrm{AE}$ generation during micromilling, considering both the mechanics of the signal generation andpropagationmechanisms. They accounted for the shear strain rate distribution on the shear plane and the dislocation density, considering a Gaussian probability density function for the distribution of AE source on the shear plane. 


\subsection{The Coroning Process}

Coroning is a complex multi-dimensional metal removal process that is used for gear fabrication. Gears finished by polishing improve functional flank topology and reduce gear noise[54]. A coroning tool and system are shown in Fig.1. It has a ring shape with teeth inside, which are coated with diamond. The tool is engaged with a gear and then rotates under pressure. In addition to the tool rotation, there is also simultaneous grinding action parallel to the rotation axis. Thus, the coroning process ensures final gear quality before its assembly in a transmission box. It has been used for transmission manufacturing, especially in volume production[54, 55]. Such a mass production process requires a real-time monitoring system to ensure quality and productivity. However, tool condition monitoring (TCM) for the coroning process has not been reported in the literature.
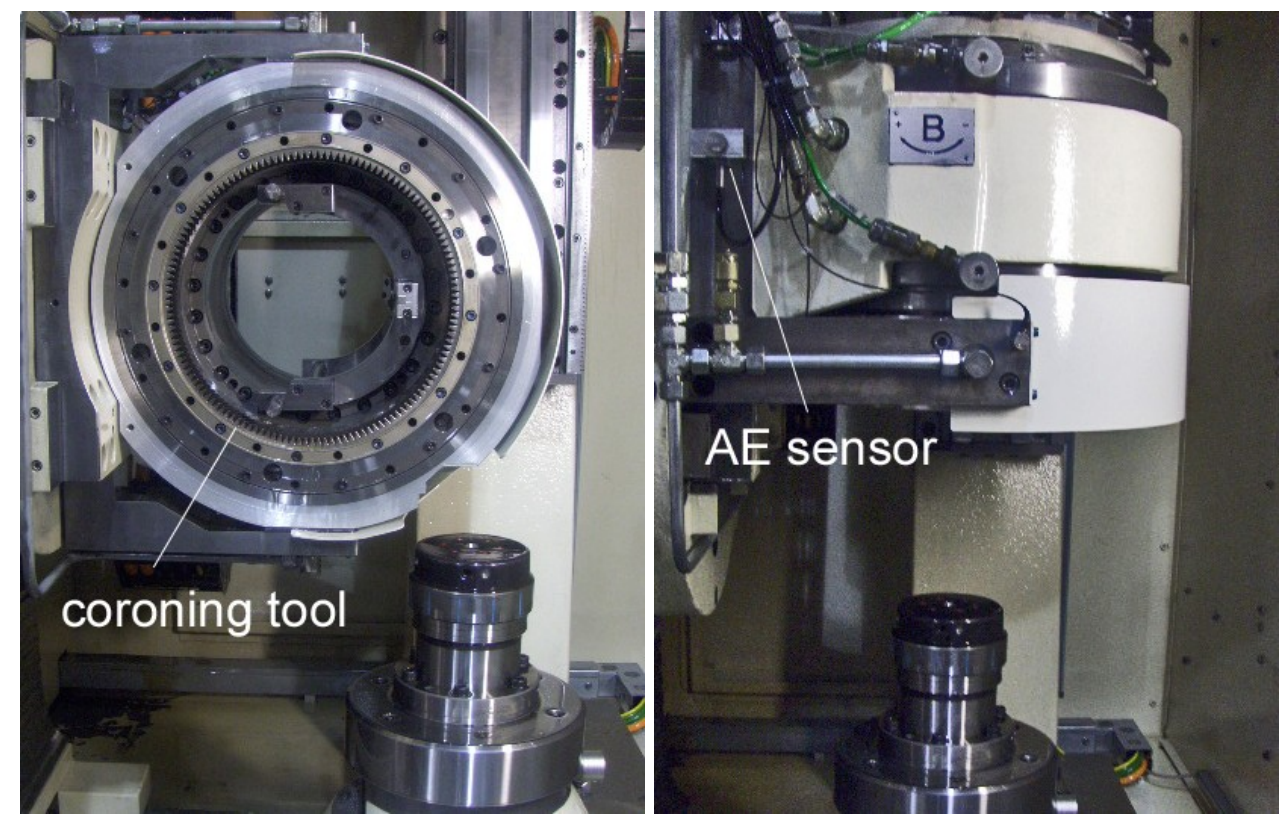

Figure 1. Coroning machine and AE sensor attachment 


\subsection{Multi-Classifier Fusion}

The fusion procedure for multiple classifiers [56-58] being considered here is illustrated schematically in Figure 2, using the hidden Markov (HMM), Bayesian rule, Gaussian mixture (GMM), and K-means models[59, 60].

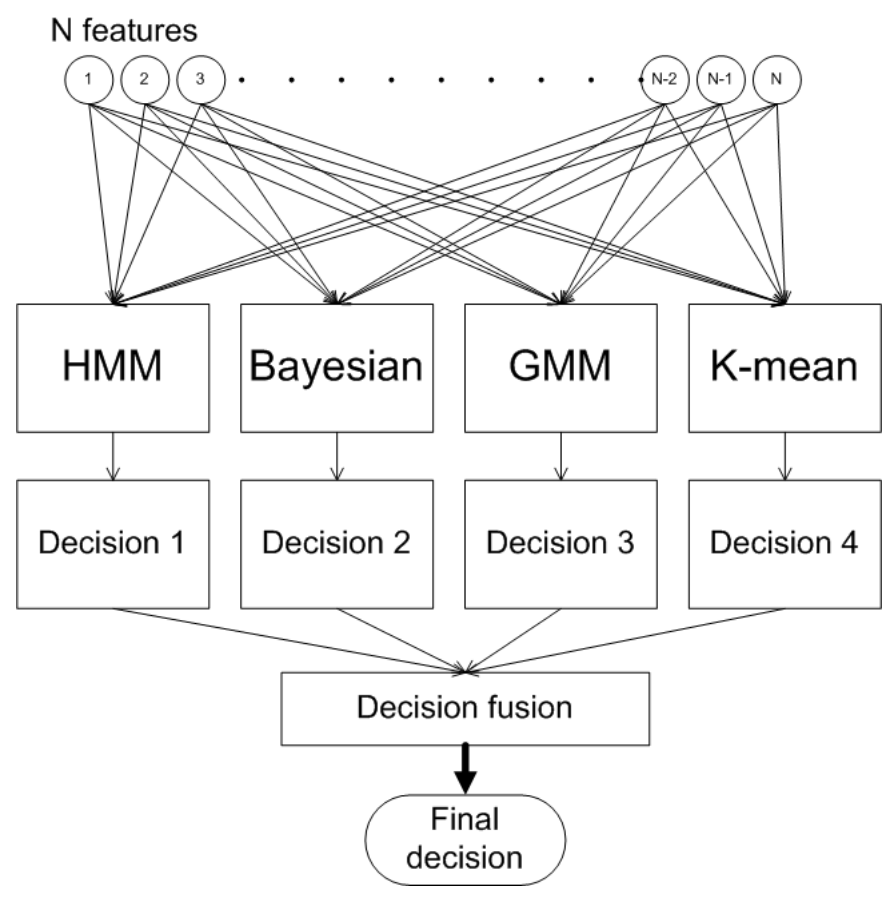

Figure 2. Multi-classifier fusion

We first define a matrix $\mathbf{B}$ consisting of the class pattern (decisions) determined by individual classifiers:

$$
\mathbf{B}=\left[\begin{array}{ccccc}
b_{1,1} & b_{1,2} & b_{1,3} & \cdots & b_{1, m} \\
b_{2,1} & b_{2,2} & b_{2,3} & \cdots & b_{2, m} \\
\vdots & & \vdots \\
b_{n, 1} & b_{n, 2} & b_{n, 3} & \cdots & b_{n, m}
\end{array}\right], \quad b_{i, j} \in\{1,2,3, \cdots, N\}
$$

where $b_{i, j}$ is the class pattern (decision) of observation $\mathrm{j}$ by classifier $\mathrm{i}$

$N$ is the number of classes or states 
$m$ is the number of observations throughout the life of the tool $n$ is the number of classifiers.

Now consider a weighting vector $\mathbf{W}$ that consists of individual classifier performances, $\mathrm{w}_{\mathrm{i}}$ :

$$
\mathbf{W}=\left[\begin{array}{lllll}
w_{1} & w_{2} & w_{3} & \cdots & w_{n}
\end{array}\right]
$$

where $w_{i}$ is the performance of classifier i.

$w_{i}$ is calculated for $\mathrm{m}$ observations as follows:

$$
w_{i}=\frac{\sum_{j=1}^{m} \delta\left(b_{i, j}, y_{j}\right)}{m} \quad \mathrm{i}=1,2, \cdots, \mathrm{n}
$$

where $y_{j}$ is the true class of observation $\mathrm{j}, \mathrm{j}=1,2,3, \ldots, \mathrm{m}$

$$
\boldsymbol{\delta}(k, l)=\left\{\begin{array}{cc}
1 & \text { if } k=l \\
0 & \text { else. }
\end{array}\right.
$$

Before the final decision takes place, let us define the voting matrix $\mathrm{V}$

$$
\mathbf{V}=\left[\begin{array}{ccccc}
v\left(b_{1,1}\right) & v\left(b_{1,2}\right) & v\left(b_{1,3}\right) & \cdots & v\left(b_{1, m}\right) \\
v\left(b_{2,1}\right) & v\left(b_{2,2}\right) & v\left(b_{2,3}\right) & \cdots & v\left(b_{2, m}\right) \\
\vdots & \vdots & \vdots & \ddots & \vdots \\
v\left(b_{n, 1}\right) & v\left(b_{n, 2}\right) & v\left(b_{n, 3}\right) & \cdots & v\left(b_{n, m}\right)
\end{array}\right]
$$

where $v(i)$ is the $\mathrm{i}^{\text {th }}$ row of the identity matrix, $\mathbf{I}$, whose rank is determined by the number of classes of interest. For example, for a 3 -class system $(\mathrm{N}=3), b_{i, j}$ is then an element of $\{1,2,3\}$, and we have a $3 \times 3$ matrix I.Elements of $\mathbf{B}, b_{i, j}$, are used to form the matrix $\mathbf{V}$. For example, $v\left(b_{i, j}\right)$ becomes $\left[\begin{array}{lll}1 & 0 & 0\end{array}\right]$ from the first row of the identity matrix, $\mathbf{I}$, when $b_{1,1}=1$. Likewise, 
$v\left(b_{n, m}\right)$ becomes $\left[\begin{array}{lll}0 & 1 & 0\end{array}\right]$ when $b_{1,1}=2$, and $\left[\begin{array}{lll}0 & 0 & 1\end{array}\right]$ when $b_{1,1}=3$. Thus the voting matrix $\mathbf{V}$ is

formed from the classifier decisions, $b_{i, j}$. The final decision equation is then the dot product of the weighting vector $\mathbf{W}$ and voting matrix $\mathbf{V}$ :

$$
\text { Final decision }=\mathbf{D}_{j}=[\underset{\text { column }}{\arg \max }[\mathbf{W} \bullet \mathbf{V}(j)]]
$$

where $\mathbf{V}(j)$ is the $\mathrm{j}^{\text {th }}$ column of the voting matrix, $\mathbf{V}$.

In the next section, we outline the experiments that were carried out to generate data for training and testing the monitoring system.

\section{EXPERIMENTS}

The AE signal was used to monitor the coroning process, using the set up shown in Fig. 1, and data was collected at a $2 \mathrm{MHz}$ sampling rate. A total of 2039 samples were collected, and approximately half of them were used as a training set for the classifiers. Additional details of the experimental set up are provided in [59]. The raw data was then converted to the frequency domain and frequency components were extracted as features. To identify a relationship between the tool condition and extracted features, the profile error of the fabricated gear was obtained by measuring profiles of gears reference to its tolerance using a coordinate measuring machine (CMM). 


\section{RESULTS AND DISCUSSION}

Figure 3 shows variation of two select features, $976 \mathrm{kHz}$ and $483 \mathrm{kHz}$, and the profile error with number of parts produced (every 200th gear sample produced during the coroning operation). Thesetwo features were selected using the class mean scatter (CMS) criterion [31, 44] which ranks features based on their ability to separate the classes of interest. The plots show good correlation between the features and the profile error, and since the profile error is primarily due to tool wear, this indicates that monitoring the tool condition using these features will give an indication of gear quality.

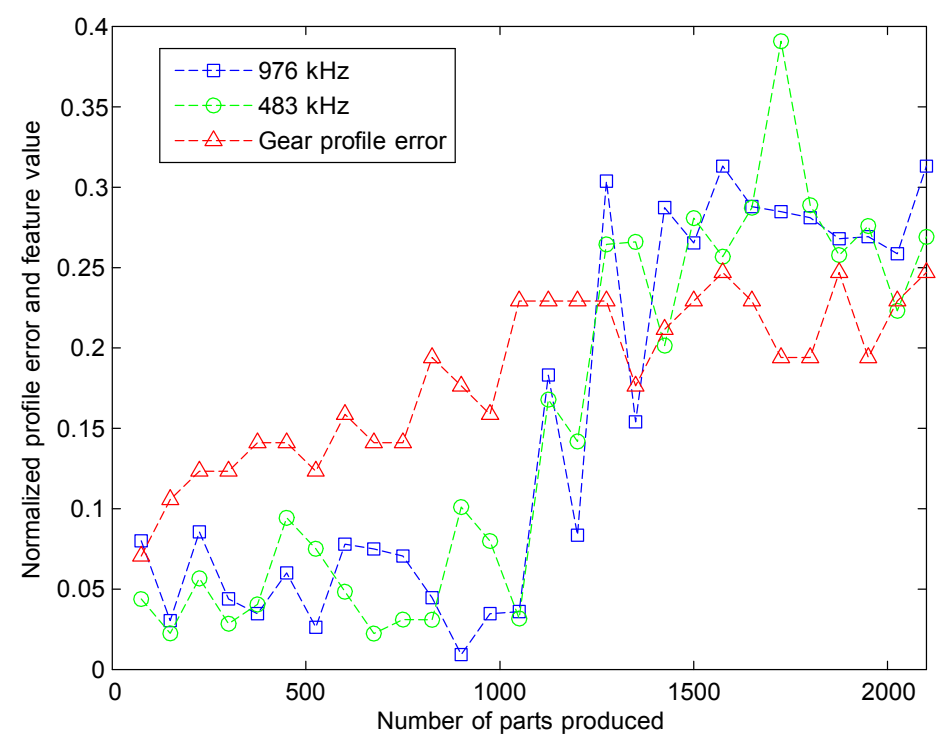

Figure 3. Profile error measured and its comparison to select features (normalized spectrum amplitude at 976 and $483 \mathrm{kHz}$ )

Since tool wear is a continuously varying process, we facilitate classification by categorizing the entire tool wear regime into three states: state 1 - sharp tool; state 2-slightly worn tool; and state 3-worn tool,Fig. 4. Figure5 shows a two dimensional feature space of these three states for two frequency features. 


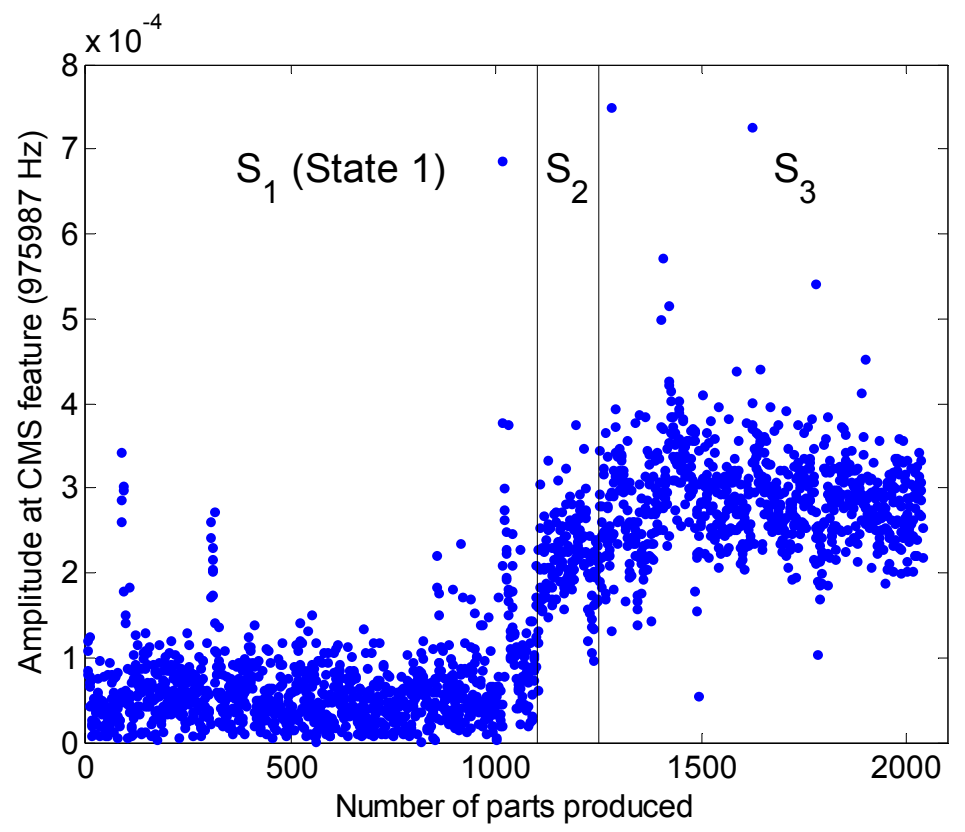

Figure 4. Variation of the AE signal amplitude at $976 \mathrm{kHz}$ with time (number of parts produced)

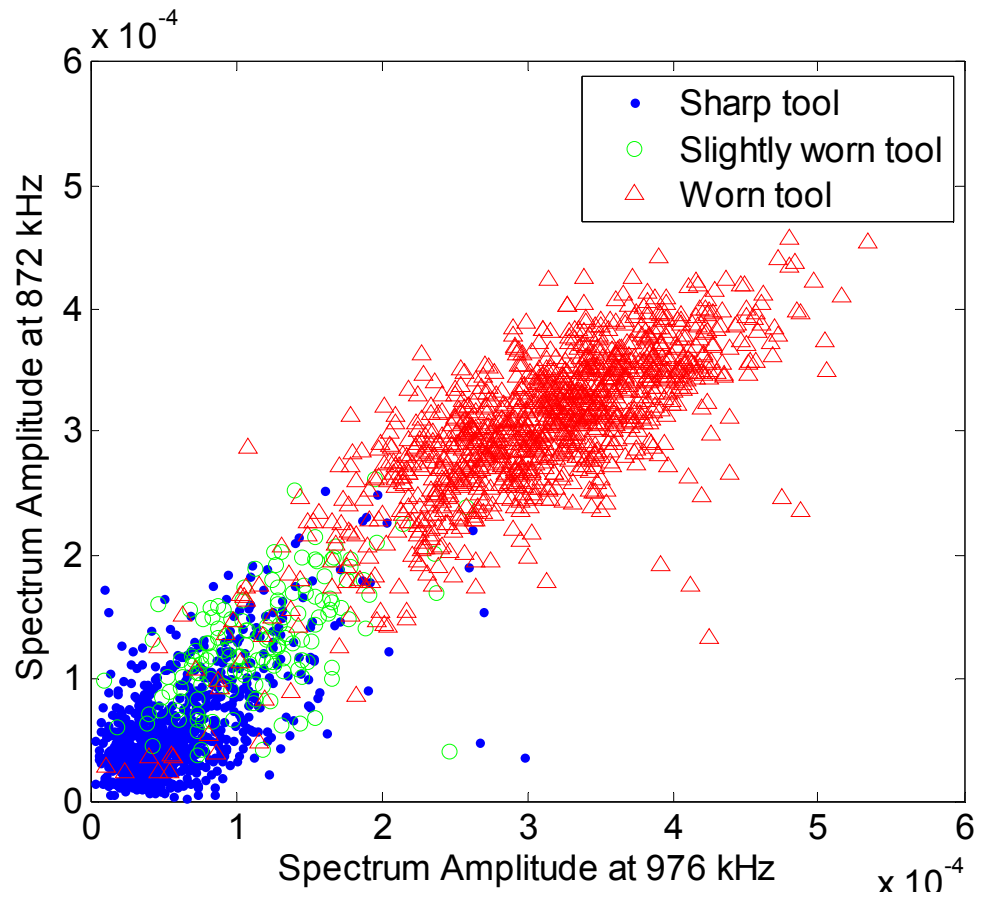

Figure 5. State of cutting tool as represented by two frequency components in the feature space 
The features selected using the class mean scatter criterion, together with the tool condition,were used for training and testing the monitoring system. The overall performances of individual classifiers for monitoring continuous tool wear of the coroning process, Figure 6, and the performances at each state,were then used as weighting factors in the classifier fusion algorithm.Theperformances of the four classifiers (hidden Markov model, minimum error rate Bayesian, Gaussian mixture model, and K-mean), were $94.1 \%, 94.1 \%, 84.0 \%$, and $67.5 \%$, respectively.

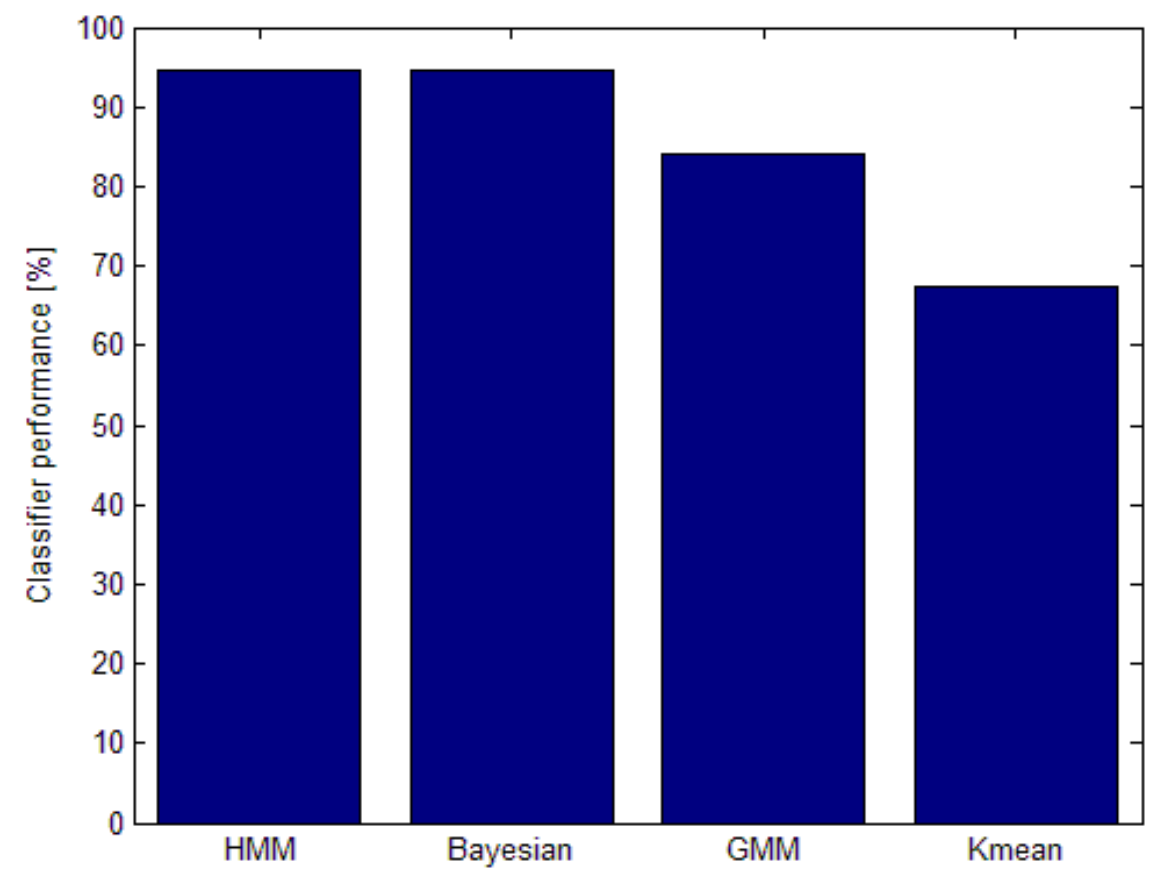

Figure 6. Overall classification rates of individual classifiers for monitoring tool wear of the coroning process

We now take an example to illustrate the influence of the weighting factor in the classifier fusion procedure outlined in section 2.3by comparing non-weighted and weighted decisions. Let us assume we have 4 classifiers, 5 observations, and 3 different states, which correspond to 
$\mathrm{n}=4, \mathrm{~m}=5$, and $b_{i, j} \in\{1,2,3\} \forall \mathrm{i}$ and $\mathrm{j}$, with unity weight values and random class patterns. Then equations (1) to (4) become:

$$
\begin{gathered}
\mathbf{W}=\left[\begin{array}{llll}
w_{1} & w_{2} & w_{3} & w_{4}
\end{array}\right]=\left[\begin{array}{llll}
1 & 1 & 1 & 1
\end{array}\right] \\
\mathbf{B}=\left[\begin{array}{lllll}
b_{1,1} & b_{1,2} & b_{1,3} & b_{1,4} & b_{1,5} \\
b_{2,1} & b_{2,2} & b_{2,3} & b_{2,4} & b_{2,5} \\
b_{3,1} & b_{3,2} & b_{3,3} & b_{3,4} & b_{3,5} \\
b_{4,1} & b_{4,2} & b_{4,3} & b_{4,4} & b_{4,5}
\end{array}\right]=\left[\begin{array}{lllll}
2 & 1 & 2 & 3 & 3 \\
1 & 1 & 2 & 2 & 3 \\
1 & 1 & 1 & 3 & 2 \\
1 & 2 & 2 & 3 & 2
\end{array}\right]
\end{gathered}
$$

And the voting matrix, $\mathrm{V}$, becomes:

$$
\mathbf{V}=\left[\left[\begin{array}{lll}
0 & 1 & 0 \\
1 & 0 & 0 \\
1 & 0 & 0 \\
1 & 0 & 0
\end{array}\right]\left[\begin{array}{lll}
1 & 0 & 0 \\
1 & 0 & 0 \\
1 & 0 & 0 \\
0 & 1 & 0
\end{array}\right]\left[\begin{array}{lll}
0 & 1 & 0 \\
0 & 1 & 0 \\
1 & 0 & 0 \\
0 & 1 & 0
\end{array}\right]\left[\begin{array}{lll}
0 & 0 & 1 \\
0 & 1 & 0 \\
0 & 0 & 1 \\
0 & 0 & 1
\end{array}\right]\left[\begin{array}{lll}
0 & 0 & 1 \\
0 & 0 & 1 \\
0 & 1 & 0 \\
0 & 1 & 0
\end{array}\right]\right]
$$

From equation $(5), \mathbf{W} \bullet \mathrm{V}(j)$ becomes;

$$
\left.\mathbf{W} \bullet \mathbf{V}(j)=\left[\begin{array}{lll}
3 & 1 & 0
\end{array}\right]\left[\begin{array}{lll}
3 & 1 & 0
\end{array}\right]\left[\begin{array}{lll}
1 & 3 & 0
\end{array}\right]\left[\begin{array}{lll}
0 & 1 & 3
\end{array}\right]\left[\begin{array}{lll}
0 & 2 & 2
\end{array}\right]\right]
$$

Thus, the final decision becomes:

$$
\text { Final decision }=\left[\begin{array}{llllll}
1 & 1 & 2 & 3 & 2 & \text { or }
\end{array}\right]
$$

In this particular example, there isa tie, as the final decision indicates. The weighting factor can play an important role in eliminating such situations by placing more emphasis on classifiers with higherweights. For example, the overall performances of individual classifiers for continuous tool wearusing the experimental data, Fig. 6,are $94.1 \%, 94.1 \%, 84.0 \%$, and $67.5 \%$ for the hidden Markov, minimum error rate Bayesian, Gaussian mixture, and K-mean models, respectively.These overall performancesare used as weighting factors as follows:

$$
\mathbf{W}=\left[\begin{array}{llll}
w_{1} & w_{2} & w_{3} & w_{4}
\end{array}\right]=\left[\begin{array}{llll}
0.941 & 0.941 & 0.84 & 0.675
\end{array}\right]
$$

Then from equation $(5), \mathrm{W} \bullet \mathrm{V}(j)$ becomes: 


$$
\mathbf{W} \bullet \mathbf{V}(j)=\left[\begin{array}{ccc}
2.456 & 0.941 & 0 \\
2.722 & 0.675 & 0 \\
0.840 & 2.557 & 0 \\
0 & 0.941 & 2.456 \\
0 & 1.882 & 1.515
\end{array}\right]^{T}
$$

resulting in the following final decision:

$$
\text { Final decision }=\left[\begin{array}{lllll}
1 & 1 & 2 & 3 & 2
\end{array}\right]
$$

which results in no more tie votes.

Since the individual classifier performances vary with the states, the classifier fusion concept can be further enhanced by updating the weighting factors using the respective classifier performancesfor each state.Three different types of weighting are investigated in this paperfor tool wear monitoring of the coroning process. First, the overall performance of each classifier is used as a weighting factor as discussed in the preceding paragraphs. State performance, in other words class dependent performance, is discussed next. Finally, penalty accommodated weighting is used, and that gives a penalty on voting for classifier fusion in order to increase reliability.

In Fig. 6, most of the error for the HMM classifier came from state 2 as Fig. 7 shows.

Table 1 summarizes the results from single classifier performance in percentages. Among the single classifiers, the HMM and Bayesian show the best overall performance. However, in state 2, the classification rateswereonly $29.3 \%$ and $38.7 \%$ using HMM and Bayesian classifiers, respectively.

Table 2 shows the results from classifier fusion with different weighting factors. 


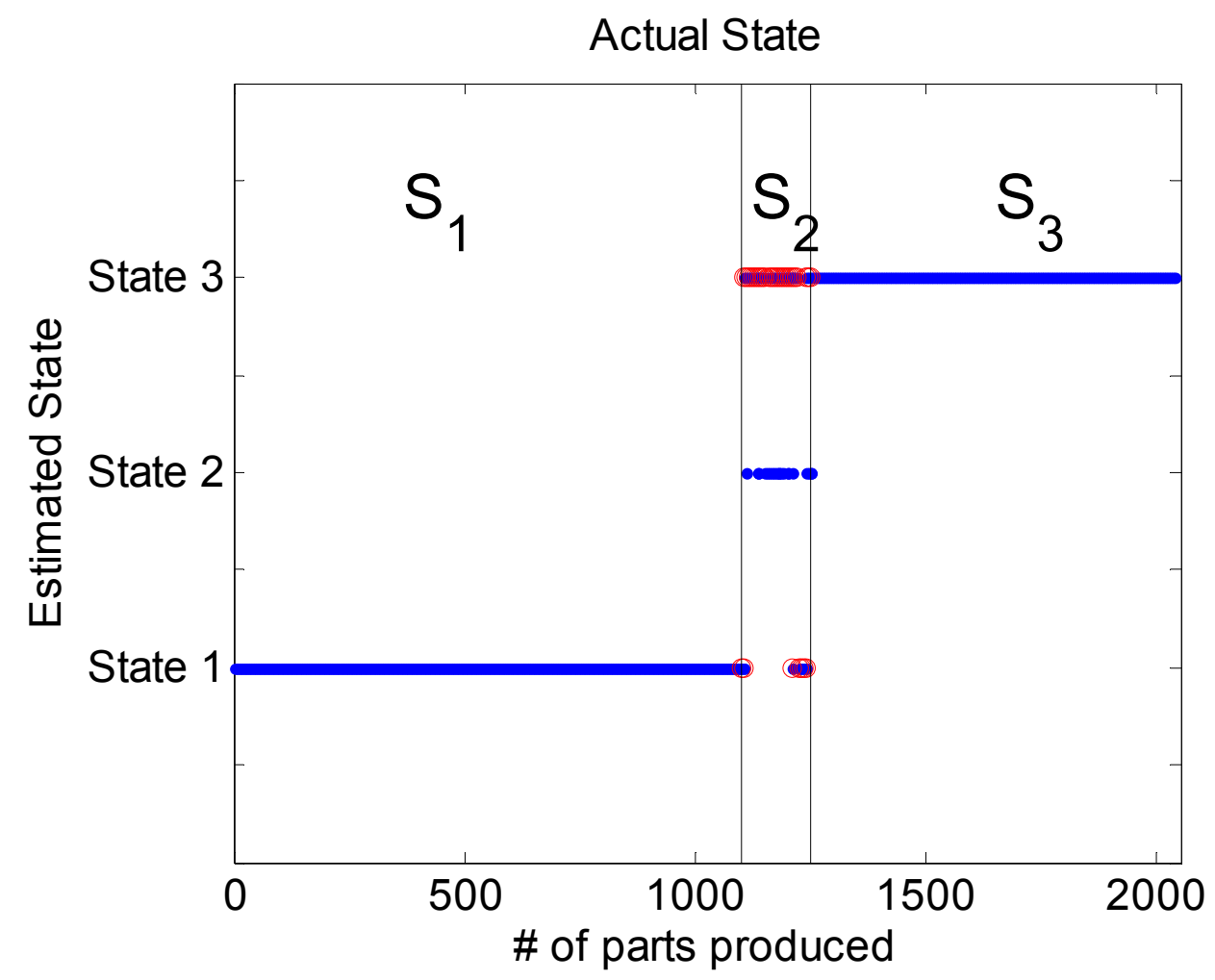

Figure 7. HMM state estimation

Table 1. Single classifier performance (\%) at each state

\begin{tabular}{|c||c|c|c|c|}
\hline & HMM & Bayesian & GMM & K-mean \\
\hline State 1 & 100.0 & 99.2 & 90.8 & 97.1 \\
\hline State 2 & 29.3 & 38.7 & 98.7 & 92.0 \\
\hline State 3 & 99.7 & 99.0 & 71.6 & 21.6 \\
\hline Overall & 94.1 & 94.1 & 84.0 & 67.5 \\
\hline
\end{tabular}

Table 2. Classifier fusion performance (\%) at each state

\begin{tabular}{|c|c|c|c|}
\hline & $\begin{array}{c}\text { Classifier fusion } \\
\text { (No weight) }\end{array}$ & $\begin{array}{c}\text { Classifier fusion } \\
\text { (Overall weight) }\end{array}$ & $\begin{array}{c}\text { Classifierfusion } \\
\text { (State weight) }\end{array}$ \\
\hline State 1 & 99.2 & 99.2 & 99.2 \\
\hline State 2 & 88.0 & 51.3 & 91.3 \\
\hline State 3 & 71.6 & 99.0 & 99.0 \\
\hline Overall & 87.7 & 95.6 & 98.5 \\
\hline
\end{tabular}


By applying the classifier fusion algorithm, the state 2 performance increased to $88 \%$ without a weighting factor, but the overall performance decreased to $87.7 \%$ compared to $94.1 \%$ without classifier fusion. Using weighted classifier fusion, with overall performance of individual classifiers as weighting factors, interestingly, the overall performance increased to $95.6 \%$ while the state 2 performance decreased to $51.3 \%$. This is because the classifier performance ispoor for the HMM and Bayesian classifiers in state 2, as Figure 8 shows, but the weight was drawn from the overall performance, Figure 6.

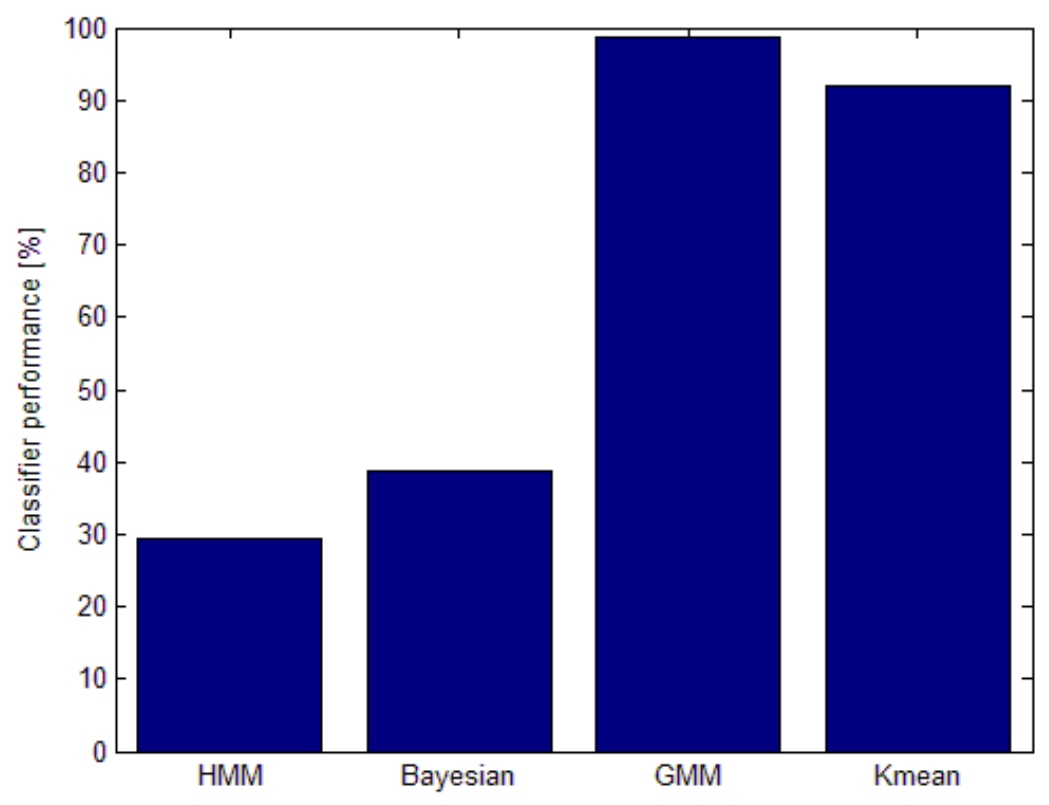

Figure 8. Classification rates of individual classifiers for monitoring the coroning process in state 2 (slightly worn tool)

Since the HMM and Bayesian classifiers had better overall performance, these were overemphasized, leadingto wrong decisionsfor state 2 . Thus it is necessary to use the performance in each state as the weighting factors. The results weresignificantly improved whenthe state weighted factors were used, 
Table 2.The average classification rate increased from $95.6 \%$ to $98.5 \%$, which is also higher than the single classifier performance of $94.1 \%$.

It is observed from Figure and Table 3that classifier fusion increased theoverall performance. However, the non-weighted classifier fusion decreased performance when compared to HMM and Bayesiansingle classifiers.

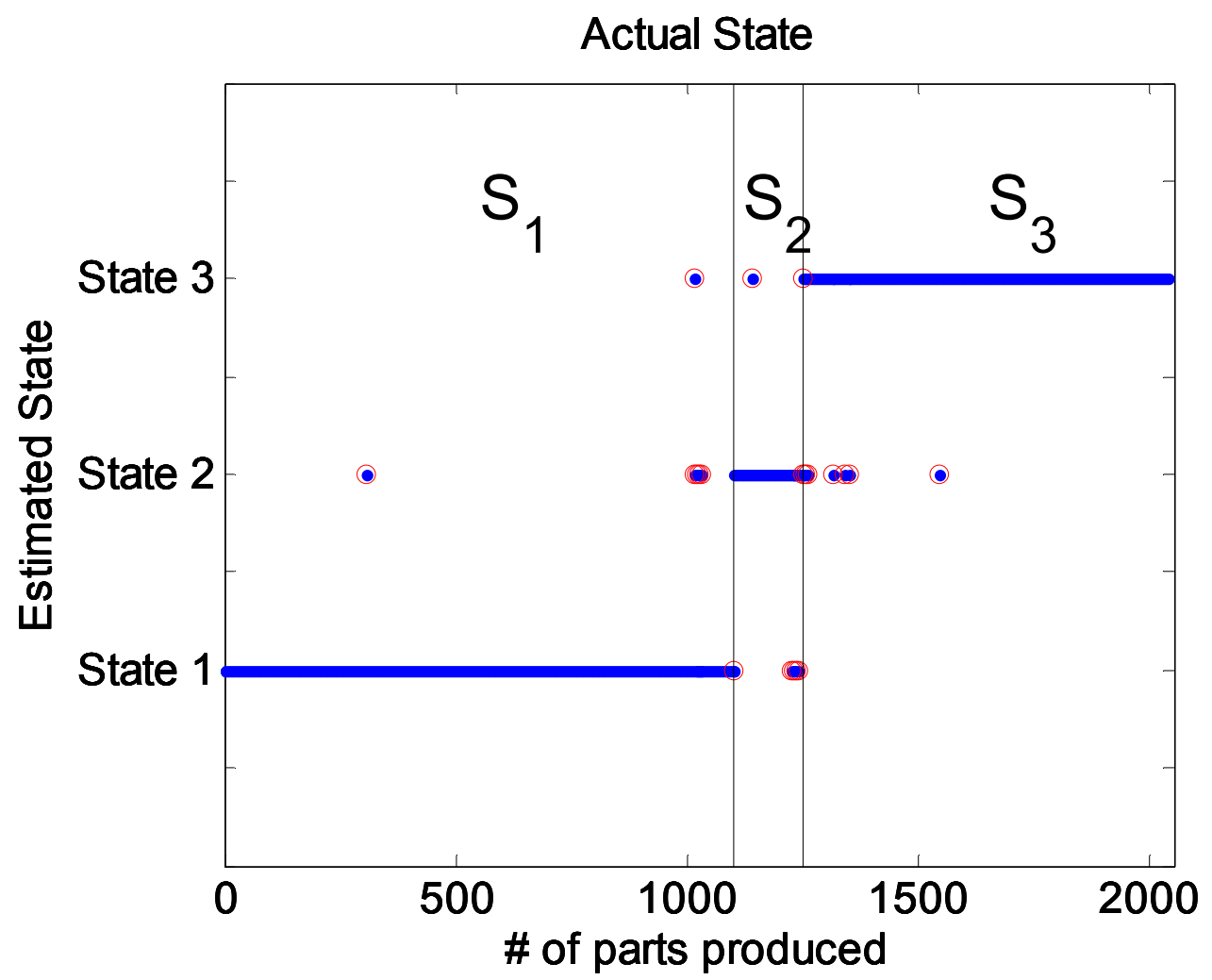

Figure 9. Classification results based on classifier fusion (state weighted majority vote)

Table 3. Single classifier vs. classifier fusion with weighted majority vote

\begin{tabular}{|c|c|c|c|c|}
\hline & HMM & Bayesian & GMM & K-mean \\
\hline Single classifier & 94.1 & 94.1 & 84.0 & 67.5 \\
\hline $\begin{array}{c}\text { Classifier fusion } \\
\text { (majority vote) }\end{array}$ & \multicolumn{4}{|c|}{87.7} \\
\hline $\begin{array}{c}\text { Classifier fusion } \\
\text { (overall weighted majority vote) }\end{array}$ & \multicolumn{4}{|c|}{95.6} \\
\hline
\end{tabular}




\begin{tabular}{|c|c|}
\hline $\begin{array}{c}\text { Classifier fusion } \\
\text { (state weighted majority vote) }\end{array}$ & 98.5 \\
\hline
\end{tabular}

State weighted classifier fusion increased overall performance, but it did not always improve the state performances. Classifier fusion with weighting factors enables more reliable decisionsas a result of thehigh performance classifiers' voting. However, ifthree out of four classifiers havelow performance, their combined effort could outweigh that of the high performing classifier, resulting in possible wrong decisions. To minimizethis possibility, an adaptive classifier fusion with weighted voting is considered. A vote penalty is incorporated into the decision, resulting in the following weighting factor:

$$
w_{f_{i}}= \begin{cases}w_{i} & \text { if } w_{i} \geq 95 \% \\ 0 & \text { otherwise }\end{cases}
$$

Substituting $w_{f_{i}}$ for $w_{i}$ gives the results shown in Figure 10 . The penalty threshold can be determined based on the manufacturer's requirements. 


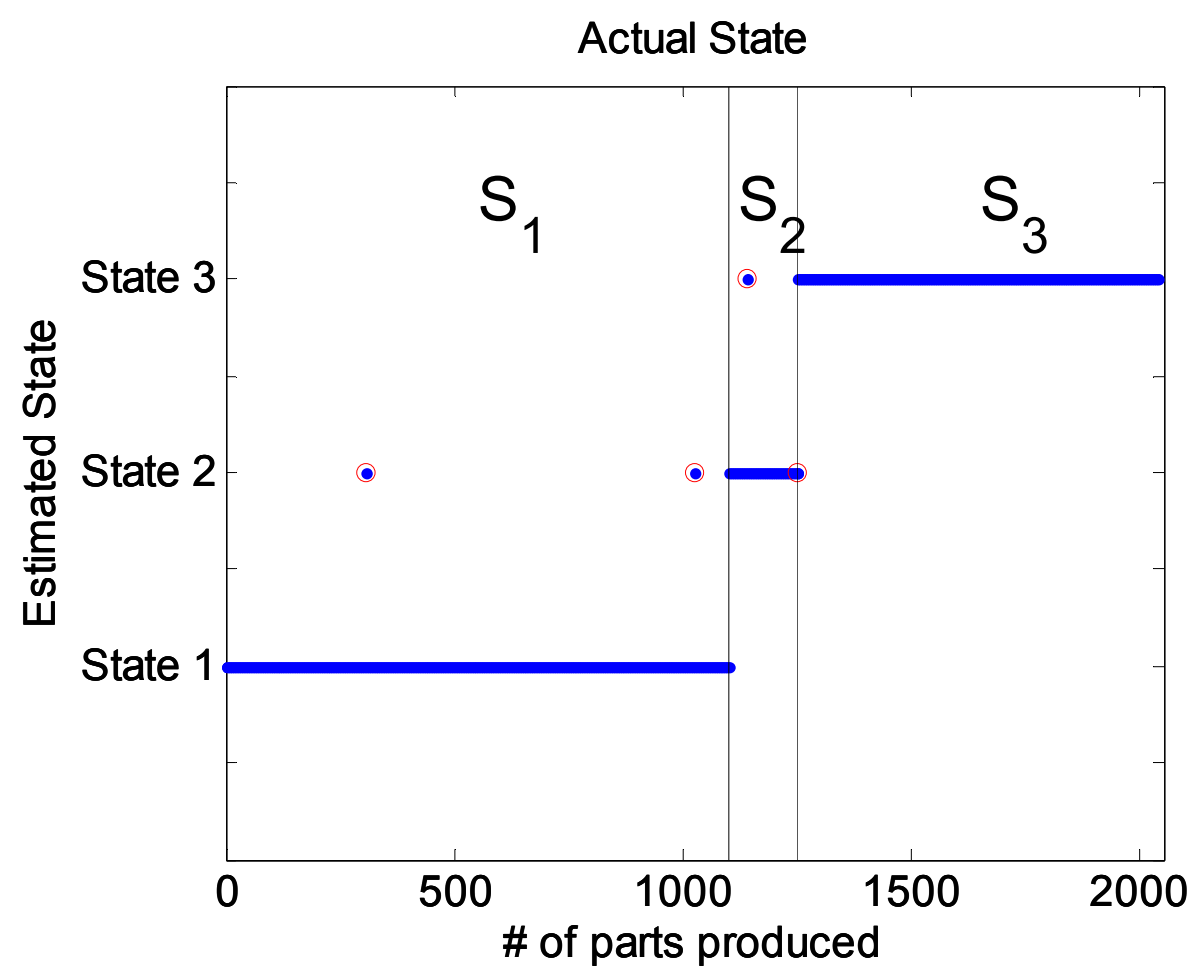

Figure 10. Adaptive classifier fusion with state weighted vote penalty Table 4. Adaptive classifier fusion compared to classifier fusion

\begin{tabular}{|c|c|c|}
\hline & $\begin{array}{c}\text { Classifierfusion } \\
\text { (State weight) }\end{array}$ & $\begin{array}{c}\text { Adaptive classifierfusion } \\
\text { (State weight vote penalty) }\end{array}$ \\
\hline State 1 & 99.2 & 99.8 \\
\hline State 2 & 91.3 & 98.7 \\
\hline State 3 & 99.0 & 99.7 \\
\hline \hline Overall & 98.5 & 99.7 \\
\hline
\end{tabular}

Table 4 shows the improvement in classification using adaptive classifier fusion with $95 \%$ vote penalty based on classifier reliability.

\section{CONCLUSIONS}

A classifier fusion algorithm adapted from decision fusion significantly increasedperformance compared to the non-fused algorithm in monitoring tool wear during 
coroning. Even though the overall monitoring performance improved with the new technique, the performance for some states improved at the expense of others. This trend was eliminated by applying an adaptive classifier fusion, where avote penalty was introduced, preventing multiple wrong votes, and this improvedclassification rate from $98.5 \%$ to $99.7 \%$.

Even though the technique was demonstrated for acoustic emission monitoring of the coroning process, it should also be applicable to any process of interest, using any suitable sensors.

\section{ACKNOWLEDGMENT}

The

authors

gratefullyacknowledge

the

financial

supportfromtheEngineeringResearchCenterforReconfigurableManufacturingSystemoftheNationa

1ScienceFoundationunderAwardNumberEEC-9529125attheUniversityofMichigan.

\section{REFERENCES}

[1] Micheletti, G.F., Koening, W., and Victor, H.R., 1976, "In-Process Tool Wear Sensors for Cutting Operations," Annals of the CIRP, 25, pp. 483-496.

[2] Tlusty, J., and Andrews, G.C., 1983, "A Critical Review of Sensors for UnmannedMachining," CIRP Annals, 32(2), p. 563.

[3] Teti, R., Jemielniak, K., O'Donnell, G., Dornfeld, D.A., 2010, “Advanced Monitoring of Machining Operations," Annals of the CIRP, 59, pp. 717-739.

[4] Leem, C. S., Dornfeld, D., and Dreyfus, S., 1995, “Customized Neural Network for Sensor Fusion in On-Line Monitoring of Cutting Tool Wear," ASME Journal of Engineering for Industry, 117(2), pp. 152-159.

[5] Kanthababu, M., Shunmugam, M. S., and Singaperumal, M., 2008, "Tool Condition Monitoring in Honing Process Using Acoustic Emission Signals," International Journal of Automation and Control, 2(1), pp. 99-112.

[6] Kannatey-Asibu, E., and Dornfeld, D.A.,1982, "A Study of Tool Wear Using Statistical Analysis of Metal Cutting Acoustic Emission,” Wear, 76, pp. 247-261.

[7] Althoefer, K., Lara, B., and Seneviratne, L. D., 2005, "Monitoring of Self-Tapping Screw Fastenings Using Artificial Neural Networks," ASME J ManufSci E, 127(1), pp. 236 245. 
[8] Dornfeld, D.A., 1990, "Neural Network Sensor Fusion for Tool Condition Monitoring," CIRP Annals - Manufacturing Technology, 39(1), pp. 101-105.

[9] Rangwala, S.S., and Dornfeld, D.A., 1989, "Learning and Optimization of Machining Operations Using Computing Abilities of Neural Networks," IEEE Transactions on Systems, Man, and Cybernetics, 19(2), pp. 299-314.

[10] Hsieh, W.H., Lu, M.C., and Chio, S.J., 2012, “Application of Backpropagation Neural Network for Spindle Vibration-Based Tool Wear Monitoring in Micro-Milling," International Journal of Advanced Manufacturing Technology, 61, pp. 53-61.

[11] Yen, C.L., Lu, M.C., and Chen, J.L., 2013, “Applying the Self-Organization Feature Map (SOM) Algorithm to AE-Based Tool Wear Monitoring in Micro-Cutting," Mechanical Systems and Signal Processing, 34, pp. 353-366.

[12] Rabiner, L. R., 1989, “A Tutorial on Hidden Markov Models and Selected Applications in Speech Recognition,” Proceedings of the IEEE, 77(2), pp. 257-286.

[13] Wang, L.,Mehrabi, M. G., and Kannatey-Asibu, E., 2002, "Hidden Markov Model-Based Tool Wear Monitoring in Machining,” ASME J ManufSci E, 124, pp. 651-658.

[14] Li, Z., Yan, X., Yuan, C., and Li, L., 2010, "Gear Multi-Faults Diagnosis of a Rotating Machinery Based on Independent Component Analysis and Fuzzy K-Nearest Neighbor," Advanced Materials Research, 108-111, pp. 1033-1038.

[15] Donat, W., Choi, K., An, W., Singh, S., and Pattipati, K., 2008, "Data Visualization, Data Reduction and Classifier Fusion for Intelligent Fault Diagnosis in Gas Turbine Engines," ASME J Eng Gas Turb Power, 130(4), pp. 1-8.

[16] Chen, L., Lu, L., Feng, K.R., Li,W., Song, J., Zheng, L., Yuan, Y., Zeng, Z., Feng, K.Y., Lu,W., and Cai, Y., 2009, "Multiple Classifier Integration for the Prediction of Protein Structural Classes," Journal of Computational Chemistry, 30(14), pp. 2248-2254.

[17] Kuncheva, L. I., 2002, “A Theoretical Study on Six Classifier Fusion Strategies,” IEEE Transactions on Pattern Analysis and Machine Intelligence, 24(2), pp. 281-286.

[18] Tolba, A. S., Khan, H. A., Mutawa, A. M., and Alsaleem, S. M., 2010, “Decision Fusion for Visual Inspection of Textiles,” Textile Research Journal, 80(19), pp. 2094-2106.

[19] Ruta, D., and Gabrys, B., 2000, “An Overview of Classifier Fusion Methods,” Computing and Information Systems, 7(1), pp. $1-10$.

[20] Sewell, M., 2011, "Ensemble Learning," http://www.academia.edu/2813351/Ensemble_learning.

[21] Altmann, A.,Rosen-Zvi, M.,Prosperi, M., Aharoni, E.,Neuvirth, H., Schulter, E.,Buch, J., Struck, D., Peres, Y.,Incardona, F.,Sonnerborg, A.,Kaiser, R.,Zazzi, M., and Lengauer, T., 2008, "Comparison of Classifier Fusion Methods for Predicting Response to Anti HIV-1 Therapy,’PLoS ONE, 3, pp. 3470-3478.

[22] Hautamäki, V., Kinnunen, T., Sedlák, F., Lee, K.A., Ma, B., and Li, H., 2013, “Sparse Classifier Fusion for Speaker Verification,"IEEE Transactions on Audio, Speech, and Language Processing, 21(8), pp. 1622-1631. 
[23] Bhattacharyya,P. and Sengupta, D.,2009, "Estimation of Tool Wear Based on Adaptive Sensor Fusion of Force And Power in Face Milling," International Journal of Production Research, 47, pp. 817-833.

[24] Nicosevici, T.,Garcia, R.,Carreras, M.,and Villanueva, M.,2004, “A Review of Sensor Fusion Techniques for Underwater Vehicle Navigation," Oceans '04 MTS/IEEE TechnoOcean '04, 3, pp. 1600-1605.

[25] Volfson, L.,2006, "Visible, Night Vision and IR Sensor Fusion," 2006 9th International Conference on Information Fusion, pp. 1-4.

[26] Muldoon, S. E.,Kowalczyk, M.,and Shen, J.,2002, "Vehicle Fault Diagnostics Using a Sensor Fusion Approach,” Proceedings of IEEE Sensors, 2, pp. 1591-1596.

[27] Sun, A.,Kannatey-Asibu, E.,and Gartner, M.,2002, "Monitoring of Laser Weld Penetration Using Sensor Fusion,” Journal of Laser Applications, 14, pp. 114-21.

[28] Subrahmanya, N. and Shin, Y.C., 2008, "Automated Sensor Selection and Fusion for Monitoring and Diagnostics of Plunge Grinding Processes,” ASMEJ ManufSci E130, 031014-1-11.

[29] Subrahmanya, N., Shin, Y.C. and Meckl, P.H., 2010, “A Bayesian Machine Learning Method for Sensor Selection and Fusion with Application to On-Board Fault Diagnostics," Mechanical Systems and Signal Processing, 24, pp. 182-192.

[30] Roli, F., Giacinto, G., and Vernazza, G., 2001, "Methods for Designing Multiple Classifier Systems," Multiple Classifier Systems. Second International Workshop, Proceedings of MCS 2001, pp. 78-87.

[31] Kannatey-Asibu, E., 2009, Principles of Laser Materials Processing, John Wiley \& Sons, New York. pp. 730-777, Chap 22.

[32] Yu, J., 2011, “Online Tool Wear Prediction in Drilling Operations Using Selective Artificial Neural Network Ensemble Model,” Neural Computing and Applications, 20(4), pp. 473-485.

[33] Ertunc, H. M., and Loparo, K., 2001, “A Decision Fusion Algorithm for Tool Wear Condition Monitoring in Drilling," International Journal of Machine Tools and Manufacture, 41(9), pp. 1347-1362.

[34] Binsaeid, S., Asfour, S., Cho, S., and Onar, A., 2008, "Machine Ensemble Approach for Simultaneous Detection of Transient and Gradual Abnormalities in End Milling Using Multisensor Fusion,” Journal of Materials Processing Technology, 209(10), pp. 47284738.

[35] Kittler, J., Hatef, M., Duin, R. P. W., and Matas, J., 1998, “On Combining Classifiers,” IEEE Transactions on Pattern Analysis and Machine Intelligence, 20(3), pp. 226-239.

[36] Kittler, J., and Alkoot, F. M., 2003, "Sum Versus Vote Fusion in Multiple Classifier Systems," IEEE Transactions on Pattern Analysis and Machine Intelligence, 25(1), pp. 110-115. 
[37] Petrakos, M., Kannelopoulos, I., Benediktsson, J. A., and Pesaresi, M., 2000, “The Effect of Correlation on the Accuracy of the Combined Classifier in Decision Level Fusion," IEEE 2000 International Geoscience and Remote Sensing Symposium, 6, pp. 2623-2625.

[38] Kahn Jr., C. E., Kalpathy-Cramer, J., Lam, C. A., and Eldredge, C. E., 2011, “Accurate Determination of Imaging Modality Using an Ensemble of Text- and Image-Based Classifiers," Journal of Digital Imaging, pp. 1-6.

[39] Hullermeier, E., and Vanderlooy, S., 2010, "Combining Predictions in Pairwise Classification: An Optimal Adaptive Voting Strategy and its Relation to Weighted Voting," Pattern Recognition, 43(1), pp. 128 - 142.

[40] Grabec, I, and Leskovar, P., 1977, “Acoustic Emission of a Cutting Process,'Ultrasonics, 15(1), pp. 17-20.

[41] Iwata, K., and Moriwaki, T., 1977, "An Application of Acoustic Emission Measurement to In-Process Sensing of Tool Wear," Annals of CRIP, 26(1), pp. 21-26.

[42] Moriwaki, T., 1980, "Detection for Cutting Tool Fracture by Acoustic Emission Measurement," Annals of CIRP, 2(1), pp. 35-40.

[43] Kannatey-Asibu, Jr E., and Dornfeld, D. A., 1981, "Quantitative Relationships for Acoustic Emission from Orthogonal Metal Cutting," ASME Journal of Engineering for Industry, 103, pp. 330-340.

[44] Emel, E.,and Kannatey-Asibu, E., 1988, “Tool Failure Monitoring in Turning by Pattern Recognition Analysis of Acoustic Emission Signals,” ASME J. Eng. for Ind., 110, pp. 137-145.

[45] Liang, S. Y., and Dornfeld, D. A., 1989, "Tool Wear Detection Using Time Series Analysis of Acoustic Emission," ASME Journal of Engineering for Industry, 111, pp. 199-205.

[46] Teti, R., 1989, "Tool Wear Monitoring Through Acoustic Emission," Annals of the CIRP, 38(1), pp. 99-102.

[47] Blum, T., and Inasaki, I., 1990, "A Study on Acoustic Emission from the Orthogonal Cutting Process," ASME Journal of Engineering for Industry, 112(3), pp. 203-211.

[48] Chiou, R.Y., and Liang, S.Y., 2000, “Analysis of Acoustic Emission in Chatter Vibration with Tool Wear Effect in Turning," International Journal of Machine Tools and Manufacture, 40(7), pp. 927-941.

[49] Dornfeld, D.A., 1992, "Application of Acoustic Emission Techniques in Manufacturing,’NDT \& E International, 25(6), pp. 259-269.

[50] Li, X., 2002, “A Brief Review: Acoustic Emission Method for Tool Wear Effect During Turning," International Journal of Machine Tools and Manufacture, 42(2), pp. 157-165.

[51] Tansel, I., Trujillo, M., Nedbouyan, A., Velez, C., Bao, W.U., Arkan, T.T., and Tansel, B., 1998, "Micro-End-Milling - III. Wear Estimation and Tool Breakage Detection Using Acoustic Emission Signals," International Journal of Machine Tools and Manufacture, 38, pp. 1449-1466. 
[52] Lee, D.E., Hwang, I., Valente, C.M.O., Oliveira, J.F.G., and Dornfeld, D.A., 2006, "Precision Manufacturing Process Monitoring with Acoustic Emission," International Journal of Machine Tools and Manufacture, 46(2), pp. 176-188.

[53] Hung, C.W., and Lu, M.C., 2013, "Model Development for Tool Wear Effect on AE Signal Generation in Micromilling," International Journal of Advanced Manufacturing Technology, 66, pp.1845-1858.

[54] Schenk, T., Uebel, U., and Wolfel, F., 2003, "Coroning: High Performance Gear Honing Without Dressing," Proceedings of the ASME Design Engineering Technical Conference, 4B, pp. 751-759.

[55] Yum, J., Kamouneh, A., Wang, W., and Kannatey-Asibu, E., 2009, “Acoustic Emission Condition Monitoring of the Coroning Process," Proceedings of the ASME International Manufacturing Science and Engineering Conference, 2, pp. 157-163.

[56] Goebel, K., and Yan, W., 2004, "Choosing Classifiers for Decision Fusion," Seventh International Conference on Information Fusion, 1, pp. 563-568.

[57] Tolba, A.S., Khan, H.A., Mutawa, A.M., and Alsaleem, S. M., 2010, "Decision Fusion for Visual Inspection of Textiles," Textile Research Journal, 80, pp. 2094-2106.

[58] Storer, M.,Urschler, M.,Bischof, H., and Birchbauer, J.A., 2008, "Classifier Fusion for Robust ICAO Compliant Face Analysis," 2008 8th IEEE International Conference on Automatic Face Gesture Recognition, pp. 2154-2162.

[59] Yum, J., Kim, T.H., and Kannatey-Asibu, E., 2013, “A Two-Step Feature Selection Method for Monitoring Tool Wear, and its Application to the Coroning Process," International Journal of Advanced Manufacturing Technology, 64(9-12), pp. 1355-1364.

[60] Duda, R.O., Hart, P.E., and Stork, D.G., 2006, Pattern Classification, John Wiley \& Sons, New York. 


\section{FIGURE CAPTIONS}

Figure 1. Coroning machine and AE sensor attachment

Figure 2. Multi-classifier fusion

Figure 3. Profile error measured and its comparison to select features (normalized spectrum amplitude at 976 and $483 \mathrm{kHz}$ ).

Figure 4. Variation of the AE signal amplitude at $976 \mathrm{kHz}$ with time (number of parts produced)

Figure 5. State of cutting tool as represented by two frequency components in the feature space

Figure 6. Overall classification rates of individual classifiers for monitoring tool wear of the coroning process

Figure 7. HMM state estimation

Figure 8. Classification rates of individual classifiers for monitoring the coroning process in state 2 (slightly worn tool)

Figure 9. Classification results based on classifier fusion (state weighted majority vote)

Figure 10. Adaptive classifier fusion with state weighted vote penalty

\section{LIST OF TABLES}

Table 5. Single classifier performance (\%) at each state

Table 6. Classifier fusion performance (\%) at each state

Table 7. Single classifier vs. classifier fusion with weighted majority vote

Table 8. Adaptive classifier fusion compared to classifier fusion 